\title{
Meta
}

Journal des traducteurs

Translators' Journal

\section{DUDUC, Robert (1991): Vocabulaire bilingue de la publicité, Montréal, Linguatech, 291 p.}

\section{Marie-Éva De Villers}

Volume 38, numéro 2, juin 1993

URI : https://id.erudit.org/iderudit/004322ar

DOI : https://doi.org/10.7202/004322ar

Aller au sommaire du numéro

Éditeur(s)

Les Presses de l'Université de Montréal

ISSN

0026-0452 (imprimé)

1492-1421 (numérique)

Découvrir la revue

Citer ce compte rendu

De Villers, M.-É. (1993). Compte rendu de [DUDUC, Robert (1991): Vocabulaire

bilingue de la publicité, Montréal, Linguatech, 291 p.] Meta, 38(2), 351-353.

https://doi.org/10.7202/004322ar

Ce document est protégé par la loi sur le droit d'auteur. L'utilisation des services d'Érudit (y compris la reproduction) est assujettie à sa politique d'utilisation que vous pouvez consulter en ligne.

https://apropos.erudit.org/fr/usagers/politique-dutilisation/
Cet article est diffusé et préservé par Érudit.

Érudit est un consortium interuniversitaire sans but lucratif composé de l’Université de Montréal, l'Université Laval et l'Université du Québec à Montréal. Il a pour mission la promotion et la valorisation de la recherche. https://www.erudit.org/fr/ 
DUDUC, Robert (1991): Vocabulaire bilingue de la publicité, Montréal, Linguatech, $291 \mathrm{p}$.

Le positionnement d'un produit, la cote brute, l'ours d'une publication demeurent des réalités mystérieuses pour la plupart des non-initiés. Est-il possible de distinguer la marque de commerce de la marque nominative, la marque de fabrique de la marque maison? Comrnent rendre en français «piggyback commercials», «prime time» ou «split run»?

Le Vocabulaire bilingue de la publicité de Robert Dubuc répond à toutes ces questions. Cet ouvrage a l'immense mérite de démontrer que, contrairement à ce que l'on croit souvent, les francophones disposent de termes parfaitement appropriés pour traduire les différentes notions de ce domaine: annonces jumelées, heures de pointe ou heures de grande écoute, tirage alterné en sont de bonnes illustrations.

Désormais, grâce à cet ouvrage bien adapté au contexte nord-américain, la «publicité effectuée subrepticement à l'occasion d'une émission de télévision [...], qui consiste à mettre une marque en évidence dans les situations présentées» n'est plus une «plug», mais une publicité clandestine. Enfin!

Il faut espérer que les publicitaires retiendront les judicieuses propositions de Robert Dubuc pour enrichir leur vocabulaire. À titre d'exemple, pour désigner le «secteur du marché visé par un produit», on pourra remplacer la «niche» omniprésente en marketing par l'équivalent qu'il suggère, créneau, puisqu'en français, la niche n'abrite que les chiens ou les statues.

Le Vocabulaire bilingue de la publicité comprend environ 2000 entrées classées dans l'ordre alphabétique de l'anglais; cet accès est le plus logique puisqu'il faut bien admettre que c'est la langue de départ du domaine. Les équivalents français sont assortis 
de définitions très concises qui situent rapidement le lecteur quant à la signification des termes traités. Les entrées sont indexées en sept sous-domaines: type de publicité, technique publicitaire, support, gestion, recherche, éthique et appellation d'emploi. Ce classement thématique est toujours très utile et très éclairant.

Cependant les profanes de la publicité resteront un peu sur leur appétit s'ils souhaitaient tout comprendre de l'univers de la publicité par cet ouvrage. Des définitions de nature un peu plus encyclopédique du type de celles du Dictionnaire de la comptabilité et des disciplines connexes de Fernand Sylvain ainsi que des renvois analogiques auraient favorisé une meilleure compréhension du domaine.

Ainsi le «marketing mix» est un concept clé du domaine sur lequel on s'interroge fréquemment. Le Vocabulaire bilingue de la publicité le définit comme suit: «Ensemble des éléments constituant la démarche mercatique d'une entreprise» et propose comme équivalent le terme marchéage. Cette définition très générale ne renseigne pas suffisamment; il apparaîtrait utile de préciser en premier lieu que le «marketing mix» est un dosage optimal entre les principaux facteurs de commercialisation et deuxièmement que ces facteurs de commercialisation sont le produit, le prix, la communication et la distribution. Bien sûr, ces précisions sont recherchées par les profanes, par les étudiants en administration notamment; elles ne sont peut-être pas essentielles pour les spécialistes ou les rédacteurs qui sont plutôt à la recherche d'une terminologie française exacte et uniforme. À cet égard, ceux-ci sont bien servis par le Vocabulaire bilingue de la publicité.

Quant à l'équivalent de «marketing mix» (proposé par arrêté au Journal officiel du 2 avril 1987), marchéage, il faut bien reconnaître qu'il n'a été repris ni par les praticiens ni par les chercheurs et spécialistes du domaine. Pour désamorcer le terme anglais qui est le seul usité, il faudra faire preuve d'imagination et de créativité.

Détail amusant, l'ouvrage accuse certaines entreprises de cannibalisme! On sait que le cannibalisme est le fait pour une personne, un animal de manger ses semblables. Le terme cannibalisme ne peut désigner le «phénomène d'effacement ou de mise à l'écart d'un ancien produit par un nouveau produit de la même marque»; il faudrait plutôt lire comme équivalent français cannibalisation.

Robert Dubuc a eu l'excellente idée d'inclure dans sa nomenclature la plupart des appellations d'emplois du domaine de la publicité : chef de création, concepteur-graphiste, directeur de produit, publicitaire conseil, représentant en publicité, etc. Le seul reproche que l'on peut formuler à propos de ces désignations de fonctions qui seront fort utiles, c'est qu'elles sont exclusivement masculines, comme c'est d'ailleurs le cas dans la plupart des ouvrages européens. Au Québec, l'appellation homme de marketing comme équivalent de marketer ne peut manquer d'étonner aujourd'hui. Sans être inexacte, elle paraît démodée.

D'un petit format pratique, le Vocabulaire bilingue de la publicité est agréable à consulter. La dimension des caractères ainsi que la disposition aérée du texte facilitent la lecture. En caractères gras, l'entrée anglaise est disposée à gauche tandis que l'équivalent français figure à droite sur la même ligne. Dans un prochain tirage, il y aurait peut-être lieu de mettre davantage en valeur les équivalents français qui sont composés en caractères maigres et qui ne se distinguent peut-être pas suffisamment de la définition qui suit. Malgré tout le soin qui manifestement a été apporté à la révision de l'ouvrage, quelques coquilles - inévitables - subsistent.

L'ouvrage comprend également un lexique français-anglais qui donne accès directement aux désignations anglaises. Enfin une bibliographie répertorie les sources techniques consultées aussi bien de langue française que de langue anglaise ainsi que les ouvrages terminologiques. 
Pour les praticiens de la publicité et du marketing, pour les professionnels de l'écriture, rédacteurs, traducteurs ou journalistes, le Vocabulaire bilingue de la publicité vient à coup sûr combler une lacune. En effet, la terminologie de ce domaine a été peu décrite jusqu'à ce jour. L'ouvrage se révèle efficace, d'accès facile, et répond à la plupart des questions linguistiques susceptibles de se poser ici au Québec. Nous ne saurions qu'en recommander vivement la consultation.

MARIE-ÉVA DE VILLERS

École des Hautes Études Commerciales, Montréal, Canada 